\title{
On Complexity of Optimal Recombination for the Travelling Salesman Problem
}

\author{
Anton V. Eremeev \\ Omsk Branch of Sobolev Institute of Mathematics, \\ 13 Pevtsov str. 644099, Omsk, Russia. \\ eremeev@ofim.oscsbras.ru
}

\begin{abstract}
Computational complexity of optimal recombination for the Travelling Salesman Problem is considered both in symmetric and in general cases. Strong NP-hardness of these optimal recombination problems is proven and solving approaches are considered.
\end{abstract}

\section{Introduction}

The Travelling Salesman Problem (TSP) is one of the well-known NP-hard combinatorial optimization problems [1]: given an $(n \times n)$-matrix $\left(c_{i j}\right)$ with nonnegative elements (distances), it is required to find a permutation $\left\langle i_{1}, i_{2}, \ldots, i_{n}\right\rangle$ of the elements $1,2, \ldots, n$ minimizing the sum $c_{i_{1}, i_{2}}+c_{i_{2}, i_{3}}+\ldots+c_{i_{n-1}, i_{n}}+c_{i_{n}, i_{1}}$. In case the matrix $\left(c_{i j}\right)$ is symmetric, the TSP problem is called symmetric as well. In case such property is not presupposed, we will say that the general case is considered.

In the general case a tour of the travelling salesman is a Hamiltonian circuit in a complete digraph without loops or multiple arcs, where the set of graph vertices is $V=\left\{v_{1}, \ldots, v_{n}\right\}$ and the set of arcs is $A$. The length of an arc $(i, j) \in A$, equals $c_{i j}$. In the symmetric case the tour direction does not matter so a travelling salesman's tour is a Hamiltonian cycle in a complete graph $G$ with the same set of vertices $V$ and a set of edges $E$, where the length of an edge $\{i, j\}$ equals $c_{i j}=c_{j i}$.

This paper is devoted to the complexity analysis of the optimal recombination problem (ORP) for the TSP. The problem consists in finding a shortest travelling salesman's tour which coincides with two given feasible parent solutions in those arcs (or edges) which belong to both parent solutions and does not contain the arcs (or edges) which are absent in both parent solutions. These constraints are equivalent to a requirement that the recombination should be respectful and gene transmitting as coined by N. Radcliffe [12].

In the symmetric case the input of ORP consists of an edge-weighted complete graph an two Hamiltonian parent cycles in it. In the general case the problem input consists of an arc-weighted directed graph and two parent circulations. In the general case, the ORP formulation implies that the direction of arcs in the desired tour must coincide with the direction of arcs in parent solutions, unless both opposite arcs between two vertices are present (in the later case both directions are possible). 
For the first time the optimal recombination was employed by C.C. Agarwal, J.B. Orlin R.P. Tai [2] for the maximum independent set problem. Presently, this approach has multiple applications. In the genetic algorithms where the set of feasible solutions is a set of permutations, the recombination procedures of such kind were used by M. Yagiura and T. Ibaraki [15], C. Cotta, E. Alba and J.M. Troya [6], W. Cook and P. Seymour [5] and D. Whitley, D. Hains and A. Howe [14]. Formulations of the optimal recombination problems in $[5,6,15]$ differ from the ORP formulation considered in this paper, e.g., in [5] the result of recombination may contain any edge belonging at least to one of the parent solutions.

Many problems, like the maximum independent set problem, admit polynomialtime recombination $[3,9]$. In a number of genetic algorithms, where the optimal recombination turns out to be NP-hard, many authors use the branch-andbound methods $[4,7]$ or dynamic programming [15] in the crossover operator. In such cases, often the approximate versions of the branch-and-bound or dynamic programming are used to avoid excessive computational cost. In dynamic programming [15], the number of states is limited by a given threshold. In the branch-and-bound methods $[4,7]$ the result of recombination is the best solution found within a limited computation time or limited number of iterations. The dimensionality of recombination problem may also be reduced by choosing an appropriate granularity of representation [6].

The paper is structured as follows. In Section 2, using the results of A. Itai, C.H. Papadimitriou and J.L. Szwarcfiter [11] we show NP-hardness of the optimal recombination problem in the symmetric case. Here we also prove the NP-hardness of optimal recombination in the general case, using the well-known idea of transforming the vertex cover problem into the TSP [1]. In Section 3 we propose reductions of the considered ORPs to the TSP on graphs with bounded vertex degrees. The resulting TSP problems may be solved, e.g. by means of the algorithms of D. Eppstein [8], which have the time bounds significantly smaller than the well-known upper bound $O\left(n^{2} 2^{n}\right)$ of the dynamic programming [10]. Concluding remarks are given in Section 4.

\section{NP-hardness of Optimal Recombination}

\subsection{Symmetric Case}

In [11] it is proven that recognition of Hamiltonian grid graphs (the Hamilton cycle problem) is NP-complete. Recall that a graph $G^{\prime}=\left(V^{\prime}, E^{\prime}\right)$ with vertex set $V^{\prime}$ and edge set $E^{\prime}$ is called a grid graph, if its vertices are the integer vectors $v=\left(x_{v}, y_{v}\right) \in \mathbf{Z}^{2}$ on plane, i.e., $V^{\prime} \subset \mathbf{Z}^{2}$, and a pair of vertices is connected by an edge iff the Euclidean distance between them is equal to 1 . Here and below, $\mathbf{Z}$ denotes the set of integer numbers. Let us call the edges that connect two vertices in $\mathbf{Z}^{2}$ with equal first coordinates vertical edges. The edges that connect two vertices in $\mathbf{Z}^{2}$ with equal second coordinates will be called horizontal edges.

Let us assume $V^{\prime}>4$, graph $G^{\prime}$ is connected and there are no bridges in $G^{\prime}$ (note that if any of these assumptions are violated, then existence of a 
Hamiltonian cycle in $G^{\prime}$ can be recognized in polynomial time). Now we will construct a reduction from the Hamilton cycle problem for $G^{\prime}$ to an optimal recombination problem for some complete edge-weighted graph $G=(V, E)$.

Let the edge weights $c_{i j}$ in graph $G$ be defined so that if a pair of vertices $\left\{v_{i}, v_{j}\right\}$ is connected by an edge of $G^{\prime}$, then $c_{i j}=0$; all other edges in $G$ have a weight 1 . Consider the following two parent solutions of the TSP on graph $G$ (an example of graph $G^{\prime}$ and two parent solutions for the corresponding TSP is given in Fig. 1).

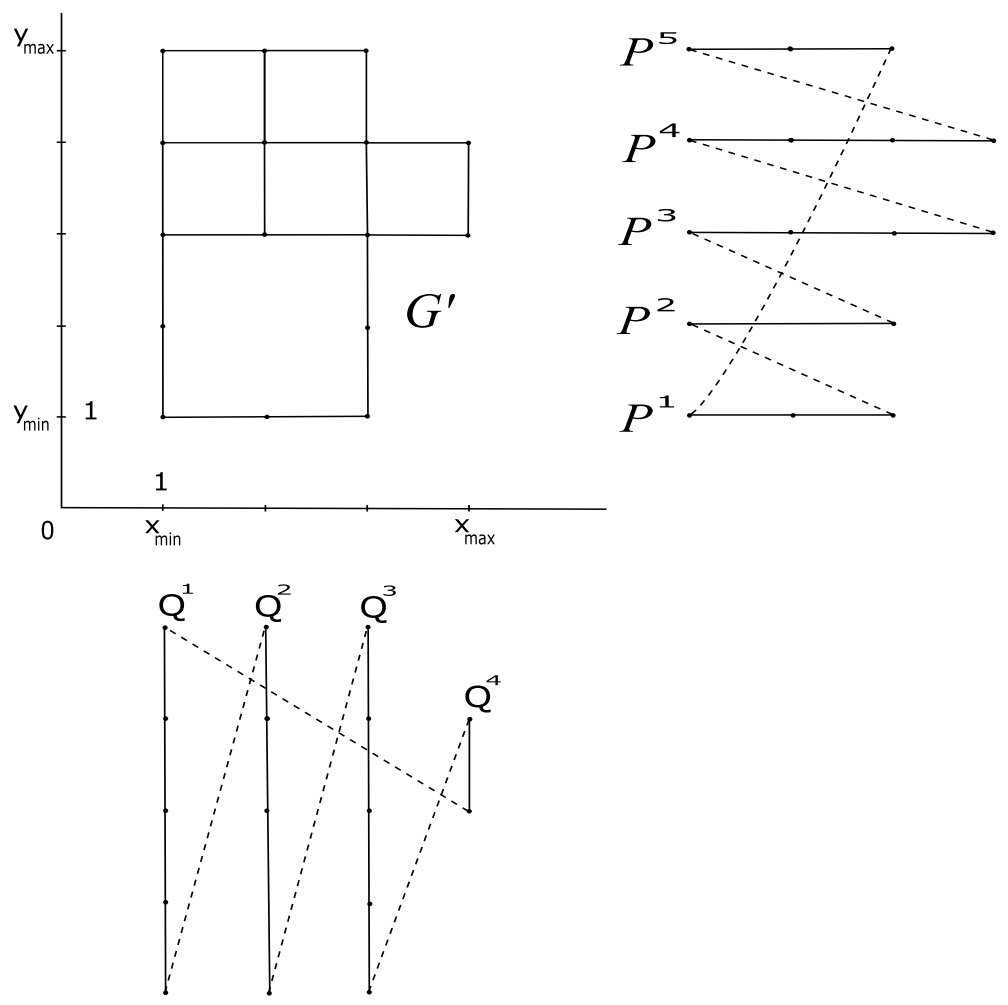

Fig. 1. Example of two parent tours used in reduction from Hamilton cycle problem to ORP in symmetric case.

Let $y_{\min }=\min _{v \in V^{\prime}} y_{v}, y_{\max }=\max _{v \in V^{\prime}} y_{v}$. For any integer $y \in\left\{y_{\min }, \ldots, y_{\max }\right\}$ denote by $P^{y}$ the horizontal chain that passes through vertices $v \in V^{\prime}$ with $y_{v}=y$ by increasing values of coordinate $x$. Let the first parent tour follow the chains $P^{y_{\min }}, P^{y_{\min }+1}, \ldots, P^{y_{\max }}$, connecting the right-hand end of each chain $P^{y}$ with $y<y_{\max }$ to the left-hand end of the chain $P^{y+1}$. Note that these connec- 
tions never coincide with some vertical edges because $G^{\prime}$ has no bridges. To create a cycle, connect the right-hand end $v_{\mathrm{TR}}$ of the chain $P^{y_{\max }}$ to the lefthand end $v_{\mathrm{BL}}$ of the chain $P^{y_{\text {min }}}$.

The second parent tour is constructed similarly using the vertical chains. Let $x_{\min }=\min _{v \in V^{\prime}} x_{v}, x_{\max }=\max _{v \in V^{\prime}} x_{v}$. For any integer $x \in\left\{x_{\min }, \ldots, x_{\max }\right\}$ let $Q^{x}$ denote the vertical chain that passes monotonically in $y$ through the vertces $v \in V^{\prime}$, such that $x_{v}=x$. The second parent tour follows the chains $Q^{x_{\min }}, Q^{x_{\min }+1}, \ldots, Q^{x_{\max }}$, connecting the lower end of each chain $Q^{x}$ with $x<$ $x_{\max }$ to the upper end of chain $Q^{x+1}$. These connections never coincide with horizontal edges since $G^{\prime}$ has no bridges. Finally, the lower end $v_{\mathrm{RB}}$ of chain $Q^{x_{\max }}$ is connected to the upper end $v_{\mathrm{LT}}$ of chain $Q^{x_{\min }}$.

Note that the constructed parent tours have no common edges. Indeed, common slanting edges do not exist since $V^{\prime}>4$. The horizontal edges belong to the first tour only, except for the situation where $y_{v_{\mathrm{RB}}}=y_{v_{\mathrm{LT}}}$ and the edge $\left\{v_{\mathrm{RB}}, v_{\mathrm{LT}}\right\}$ of the second tour is oriented horizontally. But if the first parent tour included the edge $\left\{v_{\mathrm{RB}}, v_{\mathrm{LT}}\right\}$ in this situation, then the edge $\left\{v_{\mathrm{RB}}, v_{\mathrm{LT}}\right\}$ would be a bridge in graph $G^{\prime}$. Therefore the parent tours can not have the common horizontal edges. Similarly the vertical edges belong to the second tour only, except for the case where $x_{v_{\mathrm{TR}}}=x_{v_{\mathrm{BL}}}$ and the edge $\left\{v_{\mathrm{TR}}, v_{\mathrm{BL}}\right\}$ of the first tour is oriented vertically. But in this case the parent tour can not contain the edge $\left\{v_{\mathrm{TR}}, v_{\mathrm{BL}}\right\}$, since $G^{\prime}$ has no bridges.

Note also that the union of edges of parent solutions contains $E^{\prime}$. Consequently, any Hamiltonian cycle in graph $G^{\prime}$ is a feasible solution of the ORP. At the same time, a feasible solution of the ORP has zero value of objective function iff it contains only the edges of $E^{\prime}$. Therefore, the optimal value of objective function in the ORP under consideration is equal to 0 iff there exists a Hamiltonian cycle in graph $G^{\prime}$. So, the following theorem is proven.

Theorem 1. Optimal recombination for the TSP in the symmetric case is NPhard in the strong sense.

In [11] it is also proven that recognition of grid graphs with a Hamiltonian path is NP-complete. Optimal recombination for this problem consists in finding a shortest Hamiltonian path, which uses those edges where both parent tours coincide, and never uses the edges absent in both parent tours. The following theorem is proved analogously to Theorem 1.

Theorem 2. Optimal recombination for the problem of finding the shortest Hamiltonian path in a graph with arbitrary edge lengths in NP-hard in the strong sense.

Note that in the proof of Theorem 2, unlike in Theorem 1, it is impossible simply to exclude the cases where graph $G^{\prime}$ has bridges. Instead, the reduction should treat separately each maximal (by inclusion) subgraph without bridges.

\subsection{The General Case}

In the general case of TSP the ORP is not a more general problem than the ORP considered in Subsection 2.1 because in the problem input we have two directed 
parent paths, while in the symmetric case the parent paths were undirected. Even if the distance matrix $\left(c_{i j}\right)$ is symmetric, a pair of directed parent tours defines a significantly different set of feasible solutions, compared to the undirected case. Therefore, the general case requires a separate consideration of ORP complexity.

Theorem 3. Optimal recombination for the TSP in the general case is NP-hard in the strong sense.

Proof. We use a modification of the textbook reduction from the vertex cover problem to the TSP [1].

Suppose an instance of a vertex cover problem is given as a graph $G^{\prime}=$ $\left(V^{\prime}, E^{\prime}\right)$. It is required to find a vertex cover in $G^{\prime}$ of minimal size. Let us assume that the vertices in $V^{\prime}$ are enumerated, i.e. $V^{\prime}=\left\{v_{1}, \ldots, v_{n}\right\}$, where $n=\left|V^{\prime}\right|$, and let $m=\left|E^{\prime}\right|$.

Consider a complete digraph $G=(V, A)$ where the set of vertices $V$ consists of $\left|E^{\prime}\right|$ cover-testing components, each of 12 vertices: $V_{e}=\left\{\left(v_{i}, e, k\right),\left(v_{j}, e, k\right)\right.$ : $1 \leq k \leq 6\}$ for each $e=\left\{v_{i}, v_{j}\right\} \in E^{\prime}, i<j$. Besides that, $V$ contains $n$ selector vertices, which we will denote by $a_{1}, \ldots, a_{n}$, and besides that, a supplementary vertex $a_{n+1}$.

Let the parent tours in graph $G$ be defined by the following two circuits (an example of a pair of such circuits for the case of $G^{\prime}=K_{3}$ is provided in fig. 2).

1. Each cover-testing component $V_{e}$, where $e=\left\{v_{i}, v_{j}\right\} \in E^{\prime}$ and $i<j$ is visited twice by the first tour. The first time it visits the vertices that correspond to $v_{i}$ in the sequence

$$
\left(v_{i}, e, 1\right), \ldots,\left(v_{i}, e, 6\right),
$$

the second time it visits the vertices corresponding to $v_{j}$, in the sequence

$$
\left(v_{j}, e, 1\right), \ldots,\left(v_{j}, e, 6\right)
$$

2. The second tour goes through each cover-testing component $V_{e}$, where $e=\left\{v_{i}, v_{j}\right\} \in E^{\prime}$ and $i<j$ in the following sequence:

$$
\begin{aligned}
& \left(v_{i}, e, 2\right),\left(v_{i}, e, 3\right),\left(v_{j}, e, 1\right),\left(v_{j}, e, 2\right),\left(v_{j}, e, 3\right),\left(v_{i}, e, 1\right), \\
& \left(v_{i}, e, 6\right),\left(v_{j}, e, 4\right),\left(v_{j}, e, 5\right),\left(v_{j}, e, 6\right),\left(v_{i}, e, 4\right),\left(v_{i}, e, 5\right) .
\end{aligned}
$$

The first parent tour connects the cover-testing components as follows. For each vertex $v \in V^{\prime}$ order arbitrarily the edges incident to $v$ in graph $G^{\prime}$ in sequence: $e^{v, 1}, e^{v, 2}, \ldots, e^{v, \operatorname{deg}(v)}$, where $\operatorname{deg}(v)$ is the degree of vertex $v$ in $G^{\prime}$. In the cover-testing components, following the chosen sequence $e^{v, 1}, e^{v, 2}, \ldots, e^{v, \operatorname{deg}(v)}$, this tour passes 6 vertices in each of the components $(v, e, k), k=1, \ldots, 6, e \in$ $\left\{e^{v, 1}, e^{v, 2}, \ldots, e^{v, \operatorname{deg}(v)}\right\}$. Thus, each vertex of any cover-testing component $V_{e}$, $e=\{u, v\} \in E^{\prime}$ will be visited by one of the two 6-vertex sub-tours.

The second tour passes the cover-testing components in an arbitrary order of edges $V_{e_{1}}, \ldots, V_{e_{m}}$, entering each component $V_{e_{k}}$ for any $e_{k}=\left\{v_{i_{k}}, v_{j_{k}}\right\} \in$ $E^{\prime}, i_{k}<j_{k}, k=1, \ldots, m$ via vertex $\left(v_{i_{k}}, e_{k}, 2\right)$ and exiting through vertex $\left(v_{i_{k}}, e_{k}, 5\right)$. Thus, a sequence of vertex indices $i_{1}, \ldots, i_{m}$ is induced (repetitions 

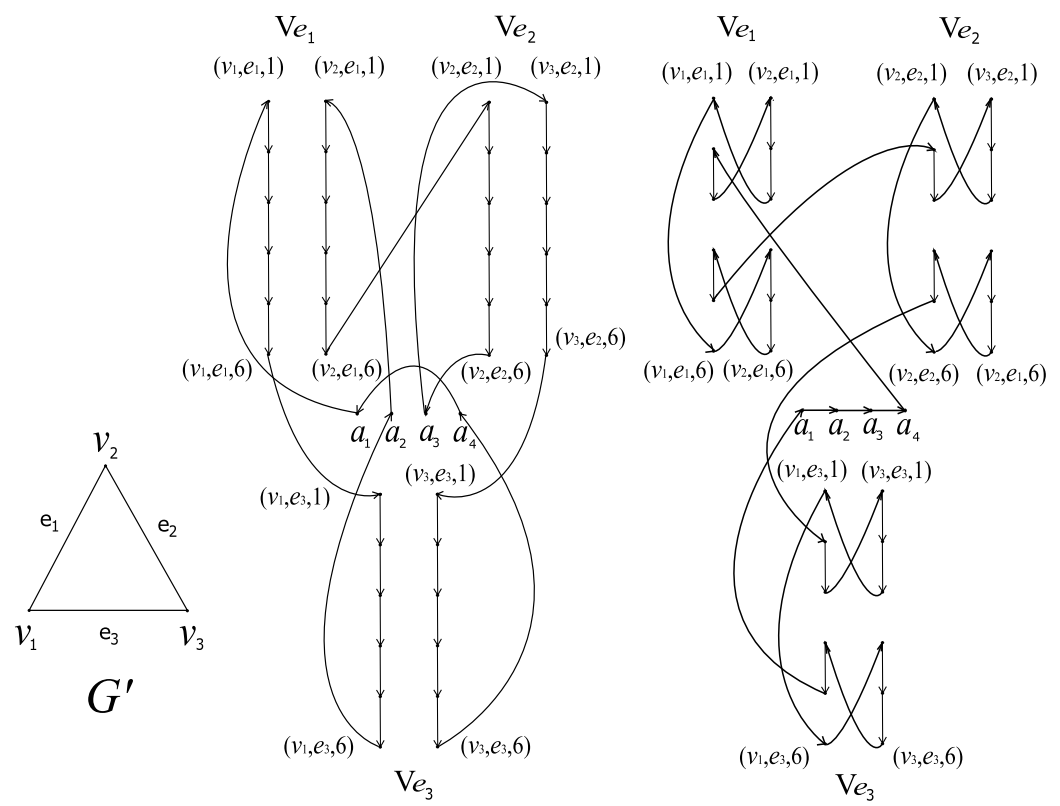

Fig. 2. A pair of parent circuits for the case of $G^{\prime}=K_{3}$. It is supposed that the incident edges are enumerated as follows. For vertex $v^{1}: e^{v_{1}, 1}=e_{1}, e^{v_{1}, 2}=e_{3}$; for vertex $v^{2}: e^{v_{2}, 1}=e_{1}, e^{v_{2}, 2}=e_{2}$; for vertex $v^{3}: e^{v_{3}, 1}=e_{2}, e^{v_{3}, 2}=e_{3}$. 
are possible). In what follows, we will need the beginning $i_{1}$ and the end $i_{m}$ of this sequence.

The parent sub-tours described above are connected to form two Hamiltonian circuits in $G$ using the vertices $a_{1}, \ldots, a_{n+1}$. The first circuit is completed using the arcs

$$
\begin{gathered}
\left(a_{1},\left(v_{1}, e^{v_{1}, 1}, 1\right)\right),\left(\left(v_{1}, e^{v_{1}, \operatorname{deg}\left(v_{1}\right)}, 6\right), a_{2}\right), \\
\left(a_{2},\left(v_{2}, e^{v_{2}, 1}, 1\right)\right),\left(\left(v_{2}, e^{v_{2}, \operatorname{deg}\left(v_{2}\right)}, 6\right), a_{3}\right), \\
\ldots, \\
\left(a_{n},\left(v_{n}, e^{v_{n}, 1}, 1\right)\right),\left(\left(v_{n}, e^{v_{n}, \operatorname{deg}\left(v_{n}\right)}, 6\right), a_{n+1}\right),\left(a_{n+1}, a_{1}\right) .
\end{gathered}
$$

The second circuit is completed by the arcs

$$
\begin{aligned}
& \left(a_{1}, a_{2}\right), \ldots,\left(a_{n-1}, a_{n}\right),\left(a_{n}, a_{n+1}\right), \\
& \left(a_{n+1},\left(v_{i_{1}}, e_{1}, 2\right)\right),\left(\left(v_{i_{m}}, e_{m}, 5\right), a_{1}\right) .
\end{aligned}
$$

Assign unit weights to all $\operatorname{arcs}\left(a_{i},\left(v_{i}, e^{v_{i}, 1}, 1\right)\right), i=1, \ldots, n$ in the complete digraph $G$. Besides that, assign weight $n+1$ to all arcs of the second tour which are connecting the components $V_{e_{1}}, \ldots, V_{e_{m}}$, the same weights are assigned to the $\operatorname{arcs}\left(a_{n+1},\left(v_{i_{1}}, e_{1}, 2\right)\right)$ and $\left(\left(v_{i_{m}}, e_{m}, 5\right), a_{1}\right)$. All other arcs in $G$ are given weight 0 .

Note that for any vertex cover $C$ of graph $G^{\prime}$, the set of feasible solutions of ORP with two parents defined above contains a circuit $R(C)$ with the following structure (an example of such circuit for the case of $G^{\prime}=K_{3}$ is provided in fig. 3).

For each $v_{i} \in C$ the circuit $R(C)$ contains the $\operatorname{arcs}\left(a_{i},\left(v_{i}, e^{v_{i}, 1}, 1\right)\right)$ and $\left(\left(v_{i}, e^{v_{i}, \operatorname{deg}\left(v_{i}\right)}, 6\right), a_{i+1}\right)$. The components $V_{e}, e \in\left\{e^{v_{i}, 1}, e^{v_{i}, 2}, \ldots, e^{v_{i}, \operatorname{deg}\left(v_{i}\right)}\right\}$ are connected together by the arcs from the first tour. For each vertex $v_{i}$ which does not belong to $C$, the circuit $R(C)$ has an $\operatorname{arc}\left(a_{i}, a_{i+1}\right)$. Also, $R(C)$ passes the $\operatorname{arc}\left(a_{n+1}, a_{1}\right)$.

The circuit $R(C)$ visits each cover-testing component $V_{e}$ by one of the two ways:

1. If both endpoints of an edge $e$ belong to $C$, then $R(C)$ passes the component following the same arcs as the first parent tour.

2. If $e=\{u, v\}, u \in C, v \notin C$, then $R(C)$ visits the vertices of the component in sequence

$$
(u, e, 1),(u, e, 2),(u, e, 3), \quad(v, e, 1), \ldots,(v, e, 6), \quad(u, e, 4),(u, e, 5),(u, e, 6) .
$$

One can check straightforwardly that this sequence does not violate the ORP constraints. 


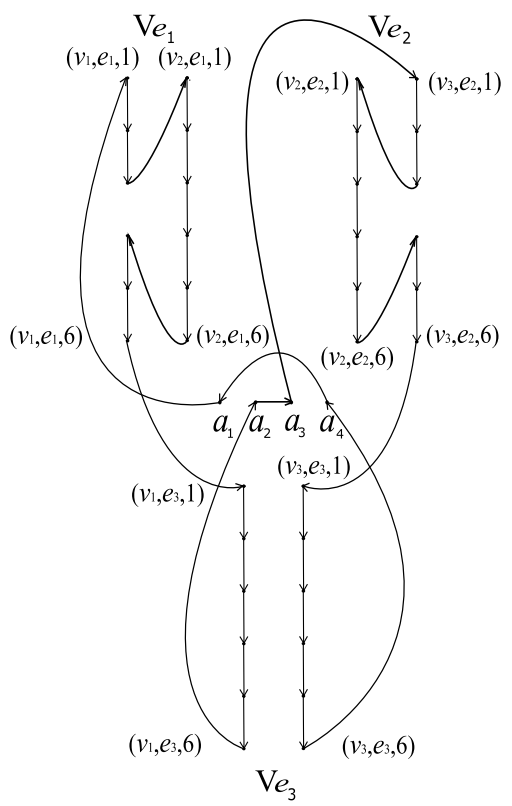

Fig. 3. An ORP solution $R(C)$ corresponding to the vertex cover $\left\{v_{1}, v_{3}\right\}$ of graph $G^{\prime}=K_{3}$. 
In general, the circuit $R(C)$ is a feasible solution to the ORP because, on one hand, all arcs used in $R(C)$ are present at least in one of the parent tours. On the other hand, both parent tours contain only the arcs of the type

$$
\begin{aligned}
& ((u, e, 2),(u, e, 3)),((u, e, 4),(u, e, 5)),((v, e, 1),(v, e, 2)), \\
& ((v, e, 2),(v, e, 3)),((v, e, 4),(v, e, 5)),((v, e, 5),(v, e, 6))
\end{aligned}
$$

within the cover-testing components $V_{e}, e=\{u, v\} \in E^{\prime}$, where vertex $u$ has a smaller index than $v$. All of these arcs belong to $R(C)$. The total weight of circuit $R(C)$ is $|C|$.

Now each feasible solution $R$ to the constructed ORP defines a set of vertices $C(R)$ as follows: $v_{i}, i \in\{1, \ldots, n\}$ belongs to $C(R)$ iff $R$ contains an arc $\left(a_{i},\left(v_{i}, e^{v_{i}, 1}, 1\right)\right)$.

Let us consider only such ORP solutions $R$ that have the objective value at most $n$. These solutions do not contain the arcs that connect the covertesting components in the second parent tour. They also do not contain the $\operatorname{arcs}\left(a_{n+1},\left(v_{i_{1}}, e_{1}, 2\right)\right)$ and $\left(\left(v_{i_{m}}, e_{m}, 5\right), a_{1}\right)$. Note that the set of such ORP solutions is non-empty, e.g. the first parent tour belongs to it.

Consider the case where the arc $\left(a_{i},\left(v_{i}, e^{v_{i}, 1}, 1\right)\right)$ belongs to $R$. Each covertesting component $V_{e}$ with $e=\left\{v_{i}, v_{j}\right\} \in E^{\prime}$ in this case may be visited in one of the two possible ways: either the same way as in the first parent tour (in this case, $v_{j}$ must also be chosen into $C(R)$ since $R$ Hamiltonian), or in the following sequence:

$$
\left(v_{i}, e, 1\right),\left(v_{i}, e, 2\right),\left(v_{i}, e, 3\right), \quad\left(v_{j}, e, 1\right), \ldots,\left(v_{j}, e, 6\right), \quad\left(v_{i}, e, 4\right),\left(v_{i}, e, 5\right),\left(v_{i}, e, 6\right)
$$

(in this case, $v_{j}$ will not be chosen into $C(R)$ ). In view of our assumption that the $\operatorname{arc}\left(a_{i},\left(v_{i}, e^{v_{i}, 1}, 1\right)\right)$ belongs to $R$, the cover-testing components $V_{e}, e \in$ $\left\{e^{v_{i}, 1}, e^{v_{i} 2}, \ldots, e^{v_{i}, \operatorname{deg}\left(v_{i}\right)}\right\}$ should be connected by the arcs of the first tour, and besides that, $R$ contains the arc $\left(\left(v_{i}, e^{v_{i}, \operatorname{deg}\left(v_{i}\right)}, 6\right), a_{i+1}\right)$. Note that the total length of the arcs in $R$ equals $|C(R)|$, and the set $C(R)$ is a vertex cover in graph $G^{\prime}$, because the tour $R$ passes each component $V_{e}$ in a way that guarantees coverage of each edge $e \in E^{\prime}$.

To sum up, there exists a bijection between the set of vertex covers in graph $G^{\prime}$ and the set of feasible solutions to the ORP of length at most $n$. The values of objective functions are not changed under this bijection, therefore the statement of the theorem follows.

\section{Transformation of the ORP into TSP on Graphs With Bounded Vertex Degree}

In this Section, the ORP problems are connected to the TSP on graphs (digraphs) with bounded vertex degree, arbitrary positive edge ( $\operatorname{arc})$ weights and a 
given set of forced edges (arcs). It is required to find a shortest Hamiltonian cycle (circulation) in the given graph (digraph) that passes all forced edges (arcs).

\subsection{General Case}

Consider the general case of ORP for the TSP, where we are given two parent tours $A_{1}, A_{2}$ in a complete digraph $G=(V, A)$. This ORP problem may be transformed into the problem of finding a shortest Hamiltonian circit in a supplementary digraph $G^{\prime}=\left(V^{\prime}, A^{\prime}\right)$. The digraph $G^{\prime}$ is constructed on the basis of $G$ by excluding the set of $\operatorname{arcs} A \backslash\left(A_{1} \cup A_{2}\right)$ and contracting each path that belongs to both parent tours into a pseudo-arc of the same length and the same direction as those of the path. The lengths of all other arcs that remained in $G^{\prime}$ are the same as they were in $G$. A shortest Hamiltonian circuit $C^{\prime}$ in $G^{\prime}$ transforms into an optimum of the ORP problem by means of reverse substitution of each pseudo-arc in $C^{\prime}$ by the path corresponding to it.

Note that there are two ingoing arcs and two outgoing arcs for each vertex in $G^{\prime}$. The TSP on such a digraph is equivalent to the TSP on a cubic digraph $G^{\prime \prime}=\left(V^{\prime \prime}, A^{\prime \prime}\right)$, where each vertex $v \in V^{\prime}$ is substituted by two vertices $\check{v}, \hat{v}$, connected by an artificial arc $(\check{v}, \hat{v})$ of zero length. All arcs that entered $v$, now enter $\check{v}$, and all arcs that left $v$ are now outgoing from $\hat{v}$. Assume that an arc $e \in A^{\prime \prime}$ is forced and called a pseudo-arc, if it corresponds to a pseudo-arc in $G^{\prime}$.

A solution to the last problem may be obtained through enumeration of all feasible solutions to the TSP with forced edges on a supplementary graph $\bar{G}=$ $\left(V^{\prime \prime}, \bar{E}\right)$. Here, a pair of vertices $u, v$ is connected iff these vertices were connected by an arc (or a pair of arcs) in the digraph $G^{\prime \prime}$. An edge $\{u, v\} \in \bar{E}$ is assumed to be forced if $(u, v)$ or $(v, u)$ is a pseudo-arc or an artificial arc in the digraph $G^{\prime \prime}$. A set of forced edges in $\bar{G}$ will be denoted by $\bar{F}$. All Hamiltonian cycles in $\bar{G}$ w.r.t. the set of forced edges may be enumerated by means of the algorithm proposed in [8] in time $O\left(\left|V^{\prime \prime}\right| \cdot 2^{(|\bar{E}|-|\bar{F}|) / 4}\right)$. Then, for each Hamiltonian cycle from $\bar{G}$ in each of the two directions we can check if it is possible to pass a circulation in $G^{\prime \prime}$, and if possible, compute the length of the circulation. This takes $O\left(\left|V^{\prime \prime}\right|\right)$ time for each Hamiltonian cycle. Note that $|\bar{E}|-|\bar{F}|=d \leq\left|E^{\prime}\right| \leq 2 n$, where $d$ is the number of arcs which are present in one of the parents only. Consequently, the time complexity of solving the ORP on graph $G$ is $O\left(n \cdot 2^{d / 4}\right)$, or $O\left(n \cdot 1.42^{n}\right)$.

Implementation of the method described above may benefit in the cases where the parent solutions have many arcs in common.

\section{$3.2 \quad$ Symmetric Case}

Suppose the symmetric case takes place and two parent Hamiltonian cycles in graph $G=(V, E)$ are defined by two sets of edges $E_{1}$ and $E_{2}$. Let us construct a reduction of the ORP in this case to a TSP with a set of forced edges on a graph with vertex degree at most 4 .

Similar to the general case, the ORP reduces to the TSP on a graph $G^{\prime}=$ $\left(V^{\prime}, E^{\prime}\right)$ obtained from $G$ by exclusion of all edges that belong to $E \backslash\left(E_{1} \cup E_{2}\right)$ and 
contraction of all paths that belong to both parent tours. Here, by contraction we mean the following mapping. Let $P_{u v}$ be a path with endpoints in $u$ and $v$, such that the edges of $P_{u v}$ belong to $E_{1} \cap E_{2}$ and $P_{u v}$ is not contained in any other path with edges from $E_{1} \cap E_{2}$. Assume that contraction of the path $P_{u v}$ maps all of its vertices and edges into one forced edge $\{u, v\}$ of zero length. All other vertices and edges of the graph remain unchanged. Let $F^{\prime}$ denote the set of forced edges in $G^{\prime}$, which are introduced when the contraction is applied to all paths wherever possible.

The vertex degrees in $G^{\prime}$ are at most 4 , and $\left|V^{\prime}\right| \leq n$. If an optimum of the TSP on graph $G^{\prime}$ with the set of forced edges $F^{\prime}$ is found, then substitution of all forced edges by the corresponding paths yields an optimal solution to the ORP problem. (Note that the objective functions of these two problems differ by the total length of contracted paths.)

The search for an optimum to the TSP on graph $G^{\prime}$ may be carried out by means of the randomized algorithm proposed in [8] for solving TSP with forced edges on graphs with vertex degree at most 4 . Besides the problem input data this algorithm is given a value $p$, which sets the desired probability of obtaining the optimum. If $p \in[0,1)$ is a constant which does not depend on the problem input, then the algorithm has time complexity $O\left((27 / 4)^{n / 3}\right)$, which is $O\left(1.89^{n}\right)$. There exists a deterministic modification of this algorithm corresponding to the case $p=1$ which requires greater computation time [8].

\section{Conclusion}

The obtained results indicate that optimal recombination for the TSP is NPhard. However, the algorithms exist that solve the optimal recombination problem in time which is significantly smaller than $O\left(n^{2} 2^{n}\right)$, the well-known time bound of the algorithm due to Held and Karp [10].

Apparently, the results on NP-hardness of the optimal recombination may be extended to some other problems, where the set of feasible solutions consists of permutations. In case the binary encoding of solutions is used, such extension could be made using the reductions of optimal recombination problems [9].

There may be some room for improvement of the algorithms, proposed in [8] for the TSP on graphs with vertex degrees at most 3 or 4 and forced edges, in terms of the running time. Thus, it seems to be important to continue studying this modification of the TSP. Also, in future it is necessary to perform experimental study of the proposed optimal recombination algorithms and compare them to other recombination methods.

\section{References}

1. Garey, M.R. and Johnson, D.S.: Computers and intractability. A guide to the theory of NP-completeness. W.H. Freeman and Company, San Francisco (1979)

2. Agarwal C.C., Orlin J.B., Tai R.P.: Optimized crossover for the independent set problem. Working paper \# 3787-95. Cambridge, MA.: Massachusetts Institute of Technology (1995) 
3. Balas E., Niehaus W.: Optimized crossover-based genetic algorithms for the maximum cardinality and maximum weight clique problems. Journal of Heuristics. 4 (2) (1998) 107-122

4. Borisovsky P., Dolgui A., Eremeev A.: Genetic algorithms for a supply management problem: MIP-recombination vs greedy decoder. European Journal of Operational Research. 195 (3) (2009) 770-779

5. Cook W., Seymour P.: Tour merging via branch-decomposition. INFORMS Journal on Computing. 15 (2) (2003) 233-248

6. Cotta C., Alba E., Troya J.M.: Utilizing dynastically optimal forma recombination in hybrid genetic algorithms. Proceedings of the 5th International Conference on Parallel Problem Solving from Nature. Lecture Notes In Computer Science, Berlin: Springer. Vol. 1498 (1998) 305-314

7. Dolgui A., Eremeev A., Guschinskaya O.: MIP-based GRASP and genetic algorithm for balancing transfer lines. Matheuristics. Hybridizing Metaheuristics and Mathematical Programming / Maniezzo, V., Stutzle, T. and Voss, S. (eds). Springer, Berlin (2010) 189-208

8. Eppstein D.: The traveling salesman problem for cubic graphs. Journal of Graph Algorithms and Applications. 11 (1) (2007) 61-81

9. Eremeev A. V.: On complexity of optimal recombination for binary representations of solutions. Evolutionary Computation. 16 (1) (2008) 127-147

10. Held, M., Karp, R. M.: A dynamic programming approach to sequencing problems. J. of Soc. for Indust. and Appl. Math. 10 (1962) 196-210

11. Itai A., Papadimitriou C. H., Szwarcfiter J. L.: Hamilton paths in grid graphs. SIAM Journal of Computing. 11 (4) (1982) 676-686

12. Radcliffe, N. J.: Forma analysis and random respectful recombination. In Proceedings of the Fourth International Conference on Genetic Algorithms. (1991) 31-38

13. Reeves C.R.: Genetic algorithms for the operations researcher. INFORMS Journ. on Comput. 9 (3) (1997) 231-250

14. Whitley D., Hains D. and Howe A.: A hybrid genetic algorithm for the traveling salesman problem using generalized partition crossover. Proceedings of the 11th International Conference on Parallel Problem Solving from Nature. Lecture Notes In Computer Science, Berlin: Springer. Vol. 6238 (2010) 566-575

15. Yagiura M., Ibaraki T.: The use of dynamic programming in genetic algorithms for permutation problems. European Journal of Operational Research. 92 (1996) $387-401$ 